\title{
Improving bowel preparation for colonoscopy in a cost-effective manner
}

\author{
Authors: Syed A Gardezi ${ }^{A}$ and Clare Tibbatts ${ }^{B}$
}

\begin{abstract}
Aims
Poor bowel preparation is the leading cause of failed colonoscopy examinations. Numerous bowel cleansing agents are in use for this purpose; however there is a noticeable limitation to their effectiveness due to variations in their tolerability and side effect profile. An inadequately prepared bowel can lead to missed diagnosis or need for repeat procedures and investigations with a significant financial impact. We reviewed the outcome of colonoscopies performed over a period of 3 months in 625 patients aiming to improve the quality of bowel preparation, and hence quality of the procedure, alongside provision of a cost-effective service.
\end{abstract}

\section{Methods}

We focused on improving the quality of bowel preparation as an indirect measure of optimal screening procedure. The secondary objective was to amend the type of preparation used and postage methods for outpatients in a cost-effective manner without compromising the quality of procedure.

Plan, do, study, act (PDSA) 1: While sending an appointment date to the patient, information leaflets were also sent along with the bowel preparation, advising patients on the optimal method of using the agent for the most favourable results, and avoiding certain foods and medications.

PDSA 2: We also noticed that money was spent on unnecessary packing while posting bowel preparation products to outpatients. These preparations were available by default in sachets quite safe for posting without using additional cardboard boxes worth $€ 5$ per postage.

PDSA 3: We moved from a practice of individual triaging to an algorithm-based system which provided more uniform prescriptions, avoiding unnecessary use of more expensive preparations.

\section{Results}

Baseline measurement: We reviewed the records of 625 colonoscopies over a period of 3 months looking particularly at the type of preparation used, quality of bowel cleansing and if

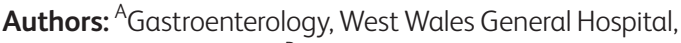
Carmarthen, Wales, UK; ${ }^{\mathrm{B}}$ Cardiff \& Vale University Health board, Cardiff, Wales, UK

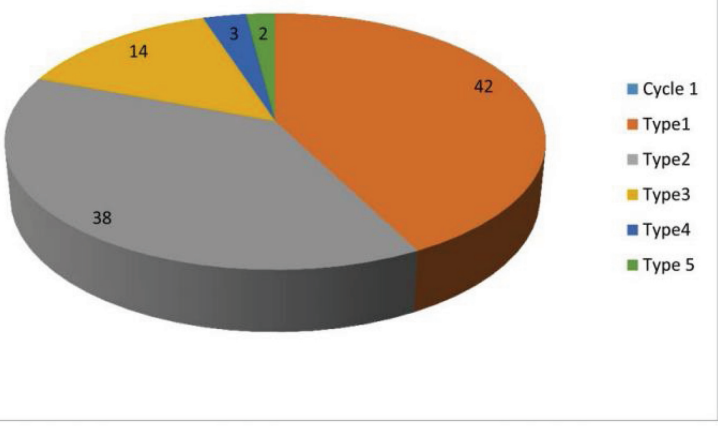

Total Patients: 625

Clear Liquids/Type 1

Cloudy Liquids/Type 2

Liquids \& Semi Solids/Type3

Semi Solids and Solids /Type 4

$232(38 \%)$

$87(14 \%)$

Solids/Type 5

$20(3 \%)$

$11(2 \%)$

Fig 1. Baseline measurements of bowel preparation quality

any repeat investigation was required due to poor cleansing. Four-hundred and fifty-five (75\%) of the patients had Kleanprep while 150 (25\%) had Moviprep, ie 100\% had polyethylene glycol (PEG) preparation. Quality of bowel cleansing was defined as clear liquids, cloudy liquids, liquids / semi-solids, semi-solids / solids, and solids and labelled as type 1-5 respectively where type 1 and type 2 were considered as adequate. Results of the first audit showed that $490(80 \%)$ patients had adequate preparation (type 1, type 2) while 118 (19\%) patients had inadequate preparation (type $3-5)$. Fourteen (2.24\%) patients needed repeat investigations either as a colonoscopy or computed tomography (CT) and six of them were brought into the hospital as an inpatient for bowel preparation on the next occasion (Fig 1).

In a repeat audit 3 months after the above interventions, we reviewed the records of 873 patients over the preceding 3 months and the results showed an improving quality of bowel preparation (Fig 2). On this occasion $83.5 \%$ (729) of patients had adequate bowel preparation while $11.60 \%$ (106) of patients had inadequate bowel cleansing for optimal assessment. Only 20 (2.29\%) patients required repeat investigations.

We re-audited the same variables after 6 months at which point all interventions had been implemented. We looked into the records of the last 100 colonoscopies and the results on 


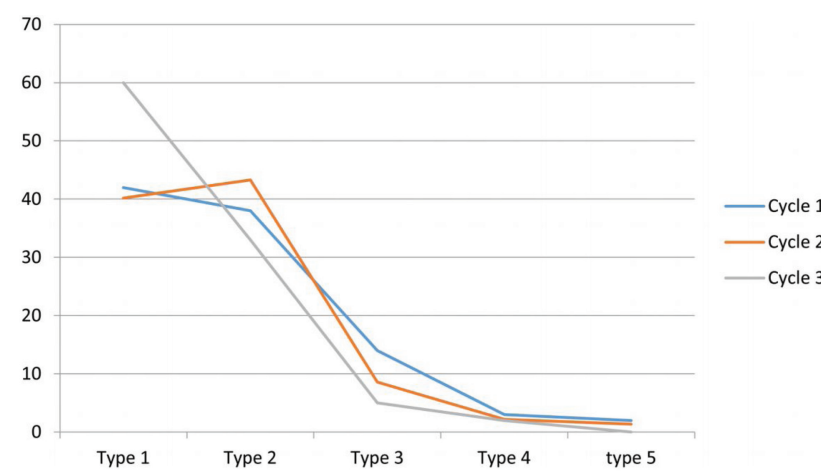

Fig 2. Comparison of three measurements

this occasion showed significant improvement from baseline measurements. Ninety-three (93\%) patients showed adequate bowl preparation while only $7 \%$ had inadequate bowel preparation.

In terms of financial implications, we estimated that based on performing 4,500 colonoscopies per year, changing the postage method alone would save $£ 23,265$ and changing prescription methods a further $£ 12,000$ (Fig 3). The biggest saving, however, would come from the reduction in number of repeat procedures required, saving an estimated $€ 150,000$ each year (Fig 4).

\section{Conclusion}

Poor bowel preparation is the leading cause of failed colonoscopies, resulting in delayed diagnosis and repeat investigations. A structured approach towards bowel preparation choice and patient education leads to better quality of bowel cleansing and is likely to improve the diagnostic yield of the procedures. This also means less numbers of repeat investigations also leading to a better financial outcome. Appropriate choice of less expensive yet equally effective bowel cleaning preparations along with their delivery to the patients can also be optimised in a much better cost-effective manner. ${ }^{1}$

\section{Conflict of interest statement}

None declared.

\begin{tabular}{|l|l|l|l|}
\hline & Moviprep & Klean Prep & $\begin{array}{l}\text { Citramag/ } \\
\text { senna }\end{array}$ \\
\hline Cost of drug & $£ 9.87$ & $£ 8.23$ & $£ 3.76$ \\
\hline $\begin{array}{l}\text { Cost of posting } \\
\text { currently }\end{array}$ & $£ 6.45$ & $£ 6.45$ & $£ 1$ \\
$\begin{array}{l}\text { Cost of posting } \\
\text { without box }\end{array}$ & $£ 1.33$ & $£ 1.33$ & \\
\hline
\end{tabular}

Fig 3. Cost of the products and their postage

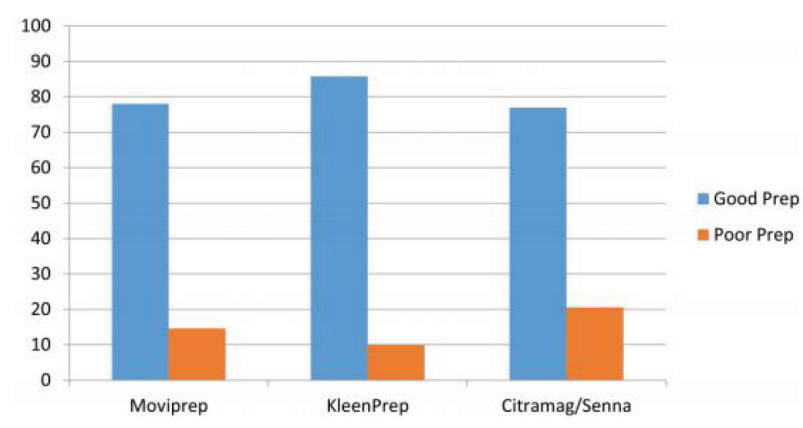

PDSA 2 Results: Total Patients 873

Type $1351(40.2 \%)$

Type $2378(43.3)$

Type $3 \quad 75(8.6 \%)$

Type $4 \quad 19(2.17 \%)$

Type $512(1.37 \%)$

PDSA 3 Results: Total Patients 100

$\begin{array}{ll}\text { Type 1 } & 60(60 \%) \\ \text { Type 2 } & 33(33 \%) \\ \text { Type 3 } & 5(5 \%) \\ \text { Type 4 } & 2(2 \%) \\ \text { Type 5 } & 0\end{array}$

Fig 4. Comparing Moviprep, Kleanprep and Citramag/senna

\section{Reference}

1 Gardezi SA, Tibbatts C. Improving bowel preparation for colonoscopy in a cost effective manner. BMJ Quality 2017;6:u204560. w5376. 